\title{
Task Dependent Synchronization of Motor Units of the Medial Gastrocnemius Muscle Revealed in EMG-Currents
}

\author{
Vinzenz von Tscharner* \\ Human Performance Laboratory, University of Calgary, Canada
}

Received: January 06, 2014; Accepted: February 25, 2014; Published: February 26, 2014

*Corresponding author: Vinzenz von Tscharner, Human Performance Laboratory, University of Calgary, Canada, Tel: +1-403-949-3714; E-mail: tvvon@ucalgary.ca

\begin{abstract}
During sport activities the gastrocnemius muscle must be activated in an orderly way to fulfill the tasks of force development, control of stability. The fascicles do not span the whole muscle and there are multiple motor units that have to be coordinated. Failure to do so results in excessive or uncoordinated forces in the joints. Thus it is important to be able to observe how synchronization of motor units changes under load. The present work is a first introduction to the possibilities of measuring the correlation of raw EMG-currents of four adjacent surface electrodes attached to the proximal region of the medial gastrocnemius muscle. In summary, the results showed and confirmed the hypotheses that the EMG-signals were correlated, that correlation was present when standing on the forefoot (tiptoe position) but increased during a repetitive calf-rising movement. The EMG-signals of adjacent electrodes could be decomposed into a reverse- and an in-phase component. The median frequencies decreased with increasing synchronization and were larger in the reverse-phase component than in the in-phase component. The results can be explained by a task dependent synchronization of motor units. Thus the method offers the possibility to study the effect of synchronization at higher levels of muscle activity.
\end{abstract}

Keywords: Electromyograms; Trans-impedance amplifier; Current amplifier; Correlation of EMG signals

\section{Introduction} Timing aspects of motor units in relation to
synchronization

Human movements have to be very tightly controlled by the neuro-muscular control mechanisms. In free style ski jumps the muscular events were as short as or shorter than $50 \mathrm{~ms}$ and inter muscular co-contractions started and were synchronized within a time range of less than $5 \mathrm{~ms}$ [1]. The Piper rhythm shows a neuromuscular control mechanism that can be seen in the EMG during isometric contractions as well as during movements [24]. It was reported that the power of the EMG signal of runners varied with the Piper Rhythm and in turn the Piper rhythm varied with running speed [5]. For the neuromuscular control to generate a Piper rhythm in the frequency range of $20 \mathrm{~Hz}$ to $50 \mathrm{~Hz}$ means that motor units (MUs) must be accurately timed and thus synchronized within a time frame of less than $50 \mathrm{~ms}$ which corresponds to higher rates of MU firing [6]. The coordinated muscle activation significantly changes in patients suffering from osteoarthritis, however, up to now, the result of this synchronization was revealed in the fluctuations of EMG-power but has not been observed in the raw EMG signal [7].

Recently [8] reviewed the MU recruitment for dynamic tasks. It was proposed that MU recruitment is likely related to the mechanical function of the muscle. Consequently they concluded that an integrative and not a reductionist approach is required to understand how recruitment is controlled during different movements. However, the recruitment was mainly studied during isometric contractions or small movements like tasks of stabilizing the body and by using indwelling electrodes that allow isolating signals of individual MUs [9]. Three processes were used to discuss the activation of the MU a) the selection of the type of MU that is required, b) the firing rate of that MU and c) the intermittent activation of the MU to adapt to short term requirements for the adjustment of balance. To adjust the muscle to the kinematics of the movement one needs an additional concept, the 'task groups' or a compartmentalization of the motor apparatus [10] and synchronization [11]. Synchronization was considered as the tendency of two MUs to fire at fixed time intervals with respect to each other more often than would be expected if the motor units fired independently and the shortterm modality was seen as a peak in the cross-interval histogram centered about zero-time delay $(0.5+/-2.9 \mathrm{~ms}$, mean +/- SD) and with an average width of $4.5+/-2.5 \mathrm{~ms}$. [11]. The short term synchronization was the dominant one. In this manuscript synchronization refers to this short term synchronization. The synchronization was expected to result in correlated EMG-signals of muscle compartments. The compartmentalization was studied using bipolar recording techniques and revealed correlations of $r^{2}$ of 0.58 for the EMG-intensity within the medial gastrocnemius but almost no correlation for the raw, bipolar EMG signal [12]. It might be that the synchronized signals were rejected by the common mode rejection of the amplifiers [13]. One could therefore expect to see correlations in monopolar measurements. 


\section{Relevance of the Anatomical aspects of the gastrocnemius medialis}

For the setup of the experiment one needs to know some anatomical properties of the medial gastrocnemius muscle. In this muscle the fascicles are attached to the upper and lower aponeuroses thus they form a penniform structure containing multiple heads structured with a large pennation angle of the fascicles [14-16]. The ultrasound images show that in the contracted form the pennation angle is almost 45 degrees and the distance between the upper and inner aponeuroses is only a few $\mathrm{cm}$. Thus fascicles that are attached to the aponeuroses under one surface electrode will be at least $20 \mathrm{~mm}$ below a second electrode that is $20 \mathrm{~mm}$ further distal of the first one and aligned in the direction of the fascicles [14]. In rat gastrocnemius endplates of distal fibers were located on the proximal third of their lengths and endplates of intermediate fibers were located at half fiber length, and for proximal fibers [17]. It is assumed that a similar, not absolutely centered distribution of locations of endplates is characteristic for the human gastrocnemius muscle which affects the timing of the extinction signals on both ends of the muscle fiber. If a conduction velocity would be calculated based on $4 \mathrm{~m} / \mathrm{s}$ one would expect the signals to be shifted by an amount that is smaller than the jitter accepted by the definition of synchronization. Based on this view of the structure of the medial gastrocnemius muscle and the expected recruitment and firing of the MUs for hypotheses were made.

\section{The four Hypotheses}

H1. The monopolar surface EMG-currents of the medial gastrocnemius muscle will be correlated while repetitively performing a calf-rising movement but also while stabilizing a forefoot tiptoe position. The reason behind this hypothesis is that the MU may be, to some extent synchronized. The extent of the synchronization is not known but is expected to be larger for the dynamic calf-rising movement than for the isometric tiptoe position.

H2. The correlation between EMG-currents of neighboring electrodes will be task dependent. For an isometric tiptoe position the MU do not need to strongly synchronize, an intermittent activation is more likely [9], however, if the task is a dynamic calfrising it has to be well timed and the MU will have to synchronize otherwise the task will not be precisely controlled.

H3. If the correlation increases and thus synchronization increases the median frequency will decrease. This hypothesis is based on theoretical predictions [18,19].

H4. If the correlation between EMG-currents of neighboring electrodes is significantly larger than 0 , then one can split the signals recorded by each electrode into an in-phase and a reverse phase component. If the in-phase component is seen as containing the part of the signal that was caused by the synchronization then its median frequency should be lower than the one of the reverse phase component.

These hypotheses follow from the assumption that muscles get activated by first selecting the right fiber type then the firing rate will be adjusted if a prolonged muscle activity is required. However, during a fast dynamic movement only a few or only one firing can occur and it is most important and most likely that these firings of different MUs occur in a synchronized, well timed process.

\section{Methods}

\section{Subjects and tasks}

Six female subjects and seven male subjects were recruited among colleagues of our research team. They were between twenty and thirty years of age and mostly well trained recreational athletes. The subjects performed first a repetitive vertical calf-rising movement e.g. a plantar flexion of the ankle joint while standing on the forefoot followed by relaxing back to a horizontal position. The up and down movement was performed at a self-selected repetition rate while standing on the edge of a step for 70s. After one minute rest, the subjects stood for 70s in a tiptoe position (maximal calf-rising position) while standing on the same edge of a step.

\section{Recording of EMG signals}

The monopolar EMG-currents (nA) were measured with a trans-impedance amplifier (bandwidth 10 to $500 \mathrm{~Hz}$ ) as described previously [13]. The trans-impedance amplifier measures the current that has to be extracted or injected into the surface electrodes $(\mathrm{Ag} / \mathrm{AgCl}$, Norotrode dual electrodes, Myotronics-Noromed Inc., Kent, WA, US) to keep the electrode at ground or reference potential. Thus all electrodes remain permanently at ground potential and therefore no lateral currents can occur. The ground electrodes, two of them, were placed on malleolus medialis and lateralis. It is important to have a high capacity ground level because all measured currents flow in and out of this ground site. Four measuring electrodes were placed in the proximal part of the medial gastrocnemius muscle. The exact location was first selected by watching ultrasound images of the muscle, later on, the location was palpated. The location of electrode 1 was $30 \mathrm{~mm}$ below the fossa popliteal and about $20 \mathrm{~mm}$ medial of the line separating the medial and lateral gastrocnemius. In this location the muscle fibers have a distinctly visible pennation angle and muscle structure seems to be well defined. The second electrode was $21 \mathrm{~mm}$ medial to the first one. The third and the forth electrodes were arranged distal to electrodes one and two, in the direction of the muscle fibers. The electrodes were mounted to the skin surface after shaving off the hairs and rubbing the surface with alcohol pads. From the recordings, a signal encompassing $60^{\prime} 000$ points $(25 \mathrm{~s})$ was selected for the analysis. The signal was filtered using a wavelet based low pass filter starting at $\mathrm{cf}=300 \mathrm{~Hz}$ with a $3 \mathrm{db}$ point at $425 \mathrm{~Hz}$ [5]. The $60 \mathrm{~Hz}$ ( 40 points per cycle) line frequency contamination was extracted from the signal by a line frequency averaging method [5].

\section{EMG analysis}

The EMG-power of the EMG-signal (25s) within the frequency range between $14 \mathrm{~Hz}$ and $500 \mathrm{~Hz}$ was computed as the sum of the power of the power spectrum in that range. The square root of the EMG-power was called EMG-intensity. The EMG-signals were then normalized, dividing by the scalar value, the EMG-intensity, 
which is equivalent to normalizing the EMG-signal to its standard deviation (STDEV). The normalized EMG-signal was called nEMG and is represented by a time series that retained the shape of the EMG signal.

\section{In summary: EMG-signal $=\mathrm{EMG}$-intensity $\bullet \mathrm{nEMG}$}

A correlation analysis was done between the nEMG signals of adjacent electrodes. A correlation was deemed not significant if it was smaller than the maximum correlation obtained by time shifting the two signals by $200 \mathrm{~ms}$ with respect to one another. The nEMG of one electrode was plotted against the nEMG of another electrode to display this correlation. The nEMG of the first electrode was plotted along the $\mathrm{x}$-axes whereas the $\mathrm{nEMG}$ of the second electrode was plotted on the y-axes.

If the results show a significant correlation, it is possible to split both nEMG signals into an in-phase and into a reverse-phase component. For this purpose the two nEMGs were arranged into two rows of a matrix(M). A $2 \times 2$ matrix (V) with elements [0.707 0.707; $0.707-0.707]$ was created. The top row of $\mathrm{V}$ represents the vector of unit length that is oriented from the lower left to the upper right in figure 2 (positive diagonal). The lower row represents a vector vertical to the first one, thus pointing from the lower right to the upper left (negative diagonal). The multiplication $\mathrm{V} \bullet \mathrm{M}$ splits the signals into the in-phase and the reverse-phase components. Just as a general comment, this is equivalent of performing a principal component analysis. Thus the power of each EMG signal is now split into an in-phase and a reverse-phase power. The ratio between the in-phase and reverse-phase power was called the axes-ratio. An axes-ratio larger than 1 means, there is more power in the in-phase part of the signal. The axes-ratio was computed for pairs of nEMGsignals recorded by adjacent electrodes. The relative decay of the axes-ratio between measurements made during the calf-rising movement and those made while standing in the tiptoe position will be reported as a percentage of the axes-ratio observed for the calf-rising movement. Both, the in-phase and reverse-phase components of the nEMG have a power spectrum with a distinct mean frequency. These mean frequencies will be compared. Power has the property of being additive. If one considers the reverse-phase power as the one associated to the nonsynchronized signal there is an equivalent power of the random EMG-signal in the in-phase direction. Therefore the power assigned to the synchronized part is the in-phase power minus the reverse-phase power and the ratio of the synchronized part to the total part was called the relative synchronized power (relsync-power) and was expressed in percentage of the total power.

The EMG-power spectra were computed by subdividing the signal into segments of 512 points and averaging these power spectra.

The statistical validation of data that were presented in the histograms were primarily based on a binomial test at the $95 \%$ level of confidence (CRITBINOM in Excel Software) whenever the null hypothesis was that the outcome was random. A correlation value was called significant if it was larger than the correlation values of time shifted signals (200 ms).

\section{Results}

\section{General observations}

The average rate of repetitive calf-rising and STDEV was 0.48 $\pm 0.17 \mathrm{~s}^{-1}$. The highest and lowest rates were $0.9 \mathrm{~s}^{-1}$ and $0.2 \mathrm{~s}^{-1}$. All measured EMG-signals were decomposed in the EMG-intensity and the nEMG. The EMG-intensity, according to the STDEV varied a lot between the four electrodes and between the 13 subjects (Table 1). The EMG-intensity of the background noise of the inactive muscle was on average $1.4 \mathrm{nA}$, however electrode 4 recorded background intensity of unknown origin of up to $3.5 \mathrm{nA}$. It might be that there is a blood vessel nearby generating charges by friction. Visual inspection of the data showed that the shape of the signals, although the amplitudes were different, were generally similar in some cases, very similar among three of the four electrodes (Figure 1). There were some cases where part of the signals seemed to change polarity. Because the EMGintensities are known to vary with the distance from the muscle fibers and depend on the fat layer under the skin the analysis of the similarity of the EMG-signals were based on the nEMG.

\section{Assessment of correlation}

A plot of $n E M G$ of one electrode against the $\mathrm{nEMG}$ of the other electrode for the electrode pairs 1-3 oriented along the fibers, 1-2 oriented in the medial lateral direction, 4-2 oriented in the direction of the fibers and 4-3 oriented in the medial lateral direction indicated that these nEMGs were correlated (Figure 2). They are visibly more correlated during the calf-rising movement (Figure 2a) than while standing in the tiptoe position (Figure 2b).

Table 1: Average EMG-intensity (nA) of male and female subjects.

\begin{tabular}{|l|c|c|c|c|c|c|c|c|}
\hline & \multicolumn{4}{|c|}{ Calf-rising ovement } & \multicolumn{4}{c|}{ Tiptoe position } \\
\hline Electrode Nr & 1 & 2 & 3 & 4 & 1 & 2 & 3 & 4 \\
\hline EMG-intensity females & 16.8 & 18.4 & 14.3 & 14.0 & 10.6 & 10.1 & 9.2 & 9.6 \\
\hline STDEV for 6 females & 6.7 & 8.7 & 6.3 & 5.2 & 4.0 & 4.6 & 3.8 & 3.3 \\
\hline EMG-intensity males & 20.5 & 23.8 & 21.9 & 15.7 & 13.3 & 15.2 & 13.2 & 8.7 \\
\hline STDEV for 7 males & 12.1 & 28.0 & 14.0 & 11.1 & 9.3 & 15.7 & 6.2 & 2.8 \\
\hline
\end{tabular}

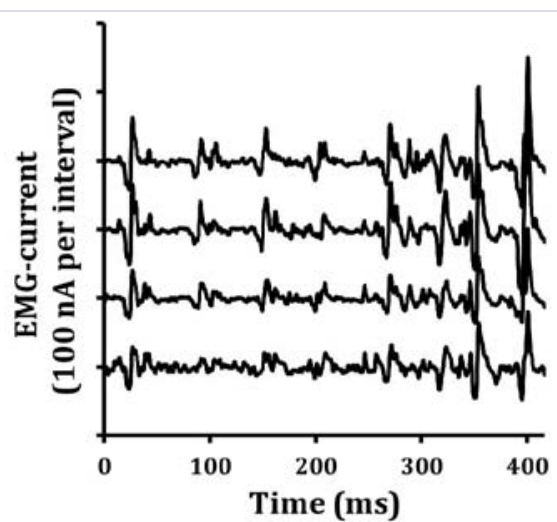

Figure 1: A sequence of $400 \mathrm{~ms}$ of the raw data during the onset of a calf-rising movement. From top to bottom the traces represent the raw EMG-currents of electrodes 1 to 4 . The lines were separated by $100 \mathrm{nA}$ for better visibility. 
The elliptic shape could also be a result of a phase shift between the pairs of nEMGs. However, we never observed a phase shift larger than $\pm 0.8 \mathrm{~ms}$. Thus the signals from all four electrodes were not phase shifted, neither in the direction of the muscle fibers nor in the medial lateral direction.

Numerically, the correlations were, for all electrode pairs, larger than the maximal correlation of the time shifted signals (0.005). The correlations between the nEMGs of the electrode pairs 1-3, 1-2 and 4-2 showed that the correlation was significantly smaller while standing in the tiptoe position than when performing the calf-rising movement (Table 2). However, the nEMG of the electrode pair 4-3, thus the medial lateral direction towards the middle of the muscle, did not reach a level of significance because of one outlier. In addition, the correlation between the electrode pair 1-3, thus electrodes that are in line with the muscle fibers, and the electrode pair 1-2 arranged in the medial lateral direction were not significantly different. On average, for the four electrode pairs and the 13 subjects, the correlation was 0.485 . The reductions of the correlations of the calf-rising movement and tiptoe position were individually different; however, they were concentrated in the range between 0.1 and 0.34 . Overall, 47 of 52 cases were positive $(\mathrm{p}<0.05)$ (Figure 3).

\section{In phase and reverse-phase decomposition}

The correlations show that the nEMGs of two electrodes can be represented as two parts, one that is a signal that is in-phase and one that is in reverse-phase between both electrodes. The axes-ratio that describes the ratio of the power between inphase and reverse-phase components of the signals indicated that the observed ratios were significantly, on average $31 \%$ lower for EMGs recorded while standing in the tiptoe position in comparison EMGs recorded while doing calf-rising movements (Figure 4).

If one assumes that the in-phase power is the sum of the reverse-phase power and a power that can be assigned to the synchronized MUs the following result for the rel-sync-power was obtained. The rel-sync-power was on average $39.5 \%$ of the total power when standing in the tiptoe position and increased to $54.2 \%$ when performing the calf-rising movement. In most cases the increase in rel-sync-power was between $10 \%$ and $40 \%$.

\section{The effect of synchronization on median frequencies}

Synchronization of MUs would theoretically result in a down shift of the power spectra. The analysis of the median frequency indicated that the average median frequency for all 104 tiptoe cases was $148 \mathrm{~Hz}$ whereas the average median frequency

\section{Calf-rising movement}
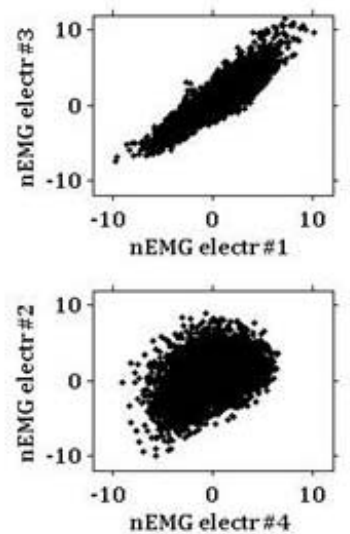
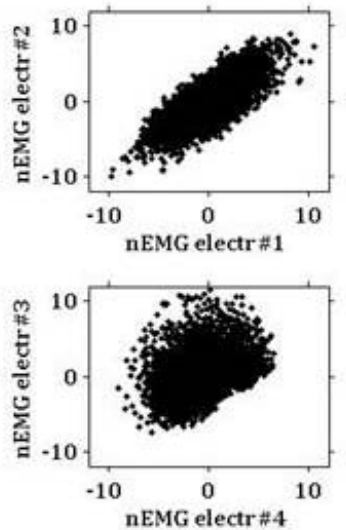

Tiptoe position
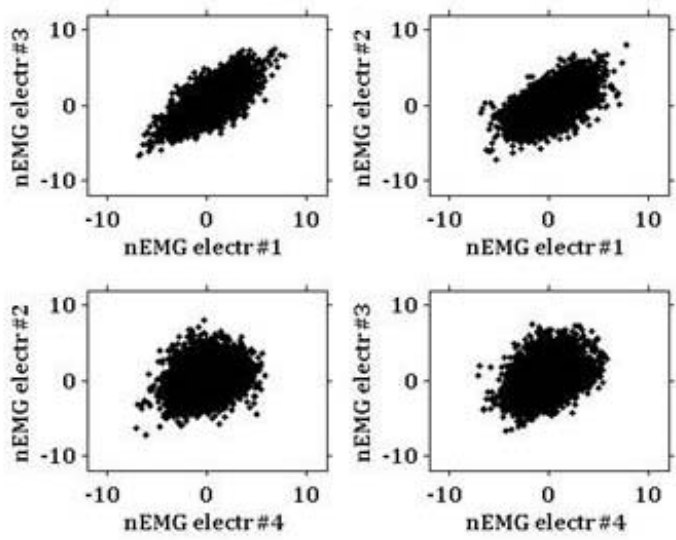

Figure 2: nEMGs of adjacent electrodes plotted against one another. The positive diagonal indicates the in-phase part of the signals and the negative diagonal the reverse-phase part. The units on the axes are STDV used to normalize the EMG-signals.

Table 2: Average correlations of male and female subjects.

\begin{tabular}{|c|c|c|c|c|}
\hline Electrode pair, $\mathrm{Nr}-\mathrm{Nr}$ & $1-3$ & $1-2$ & 4-2 & 4-3 \\
\hline Correlations while calf-rising & 0.601 & 0.596 & 0.460 & 0.283 \\
\hline Correlations for tiptoe position & 0.523 & 0.503 & 0.287 & 0.222 \\
\hline Difference & $0.078^{*}$ & $0.094^{*}$ & $0.173^{*}$ & 0.061 \\
\hline STDEV of differences & 0.037 & 0.023 & 0.030 & 0.055 \\
\hline$t$-value for 13 subjects & 2.112 & 4.073 & 5.814 & 1.106 \\
\hline $\mathrm{p}$-values one sided & 0.027 & $<0.01$ & $<0.01$ & 0.140 \\
\hline
\end{tabular}

Notes. Differences labeled with * are significant with the indicated p-values.

Difference 4-3 was not significant because of one outlier.

The value would be $0.113(\mathrm{p}=0.018)$ without the outlier. 
observed during the calf-rising movement was $122 \mathrm{~Hz}$ thus $26 \mathrm{~Hz}$ lower. In fact, out of the 104 cases, a significant number of 98 cases, showed a lower median frequency while doing the calfrising movement (Figure 5).

The analysis of the power spectra of the in- and reverse-phase signals showed that for both, the calf-rising movement and tiptoe position of all subjects and all electrode pairs (104 cases) the median frequency was higher in the reverse phase components. The average median frequency for the in-phase signals was $123 \mathrm{~Hz}$ whereas the one of the reverse phase signals was $147 \mathrm{~Hz}$. Thus the in-phase signals had a $23 \mathrm{~Hz}$ lower median frequency. In fact, a significant amount of 88 out of 104 cases showed a lower median frequency for the in-phase component (Figure 6).

\section{Discussion}

\section{Confirmation of the hypotheses}

When measuring EMG-currents the potentials cannot build up at the surface of the skin under the electrodes [5]. As a result there are no lateral currents flowing along the surface of the skin. This new method clearly showed that the signals of neighboring electrodes in the proximal part of the medial gastrocnemius muscle were correlated thus confirming hypothesis H1. The correlations were larger for the calf-rising movement than while standing in a tiptoe position thus confirming H2. As expected, synchronization leads to lower mean frequencies as postulated

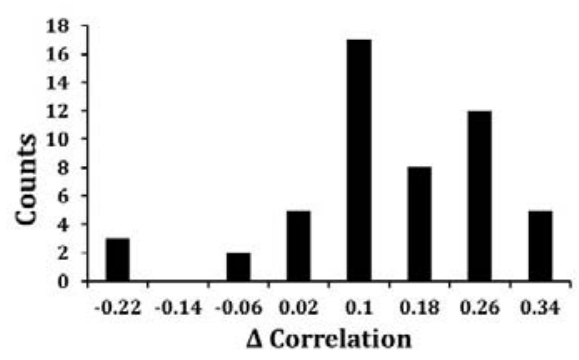

Figure 3: Histogram of the correlations measured while calf-rising minus the correlations measured in tiptoe position (bin width 0.08). The null hypothesis that the $\Delta$ correlation was randomly distributed between positive and negative values was rejected with $\mathrm{p}>0.95$.

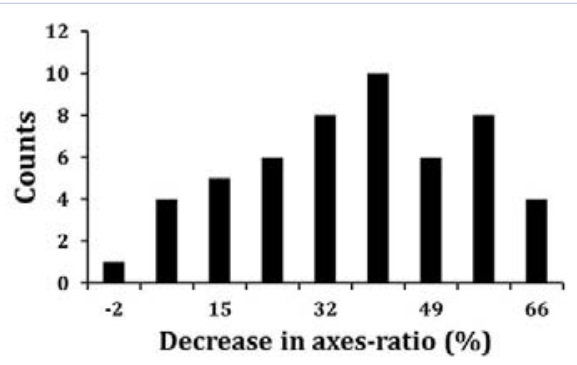

Figure 4: Histogram of the relative decrease of the axes-ratio (EMG-intensity in-phase divided by EMG-intensity reverse-phase) from the calfrising movement to the tiptoe position. (13 subjects and four electrode pairs thus for 52 cases). The null hypothesis that the decrease in axesratio was randomly distributed between positive and negative values was rejected with $\mathrm{p}>0.95$.

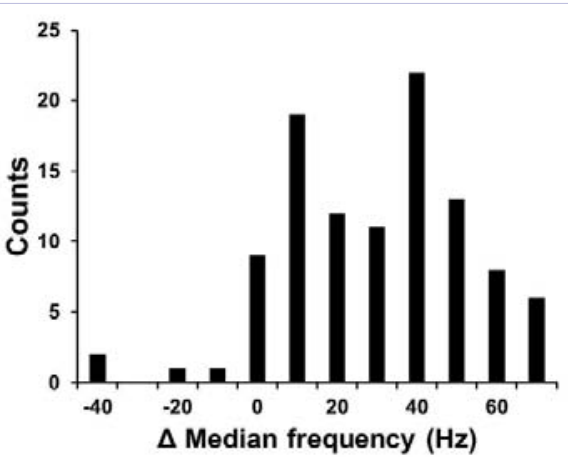

Figure 5: Histogram of the absolute decrease of the median frequency when changing from the calf-rising movement to the tiptoe position. (13 subjects, four electrode pairs for the in- and reverse-phase signals, thus 104 cases). The null hypothesis that the $\Delta$ median frequency was randomly distributed between positive and negative values was rejected with $p>0.95$.

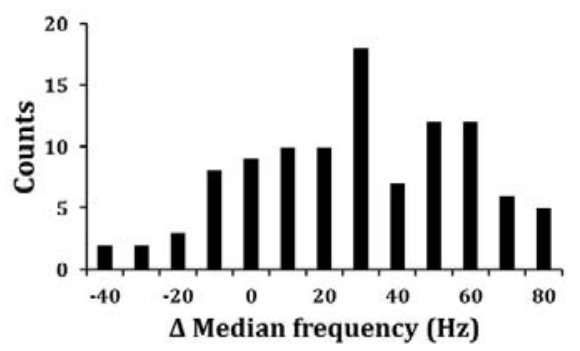

Figure 6: Histogram of the absolute difference of the median frequency between the in- and reverse-phase signals. (13 subjects and four electrode pairs for calf-rising movement and tiptoe position, thus 104 cases). The null hypothesis that the $\Delta$ median frequency was randomly distributed between positive and negative values was rejected with $p$ $>0.95$.

in H3. It was possible to split the signal into an in-phase and a reverse phase component whereby the in-phase component contains most of the synchronized signal and thus has a lower median frequency, thus confirming $\mathrm{H} 4$.

\section{Loss of bipolar EMG-potentials caused by synchronization}

Colleagues are rightfully very skeptical towards the new technology and the fact that significant correlations were observed. However, the same skepticism has to be applied when interpreting results from EMG-potential measurements. With respect to the effect of synchronicity of MUs, and to the best of our knowledge, no article pointed to the fact that bipolar EMG measurements would suppress signals generated by synchronized MUs, thus MUAP that would yield correlated EMGsignals at the electrodes of a bipolar measurement setup. The correlated signals, thus those resulting from synchronization are eliminated by the common mode rejection or at least disrupted in an unspecific way if the amplitudes are not identical. What can be observed with a bipolar setup is the reverse-phase component only. It is therefore understandable that previous bipolar measurements revealed no significant correlation of the raw 
signals although the EMG-intensity (square root of instantaneous power) was correlated [12].

In all our cases the signals between adjacent electrodes were correlated and one can therefore extract the reverse-phase part of the signal, the part that would be measured in a bipolar setup. It is understandable that, as shown in Figure 2, sufficient EMG-power was present in the reverse-phase component of the EMG-signal to detect the variations of muscle activity. However, the reversephase component changed with the degree of synchronization and thus with the task. Therefore the power of the reverse-phase varies not only as a response to an altered muscle activity but also in response to the complexity (dynamic versus isometric) of the task.

\section{Critical interpretation of the EMG measurements on the gastrocnemius muscle}

Although most people are aware that the gastrocnemius muscle has a penniform structure of the fascicles, it is still common practice to align the electrodes along the muscle fibers. One does keep a blind eye on the fact that the fibers that cross the first electrode at a close distance are far away from the second electrode and therefore add a different contribution to the current or potential to each of the electrodes [20]. Thus the difference in the EMG-signals of the electrode pairs 1-3 and 4-2 are not an approximation of the differentials of the contributing MUAPs. In fact, the results revealed no phase shift larger than $0.8 \mathrm{~ms}$ and thus did not indicate that we observed time shifted signals from the same muscle fibers.

\section{Are MUs under the different electrodes independently controlled?}

According to model calculations a significant part of the EMGsignal is caused by the terminal phase [21]. The terminal phase is strongest where the muscle fibers are attached to the outer aponeuroses, thus closest to the electrodes. If we assume that the signals were dominated by the terminal phase then one has to conclude that the correlation is caused by activating different muscle fibers of the same MU or by synchronizing the activation of the MUs. If muscle fibers belonging to the same MU were present under adjacent electrodes, or if there was cross talk between the electrodes, the correlation would be the same for both kinds of movements. Because the correlation and the axes-ratio changed (Figure 4) one can conclude that the MUs under the different electrodes were independently controlled by the nervous system. The larger correlations and decreased axes-ratio observed while calf-rising indicated that a higher synchronization was required when the timing of the task had to be more precise. A previous study showed that when the medial gastrocnemius muscle was isometrically contracted, and no additional stabilizing task was required, the inter electrode coherence was very low [5]. The results thus confirm our second hypothesis that correlation and thus synchronization of MUs are task specific. If the task is limited to stabilizing the human body only intermittent activities are necessary and it is likely that they too are synchronized across the whole muscle [15].

\section{Shift of median frequency caused by synchronization}

As predicted theoretically and often stated thereafter, the median frequency will shift to lower frequencies when MUs get synchronized $[18,19]$. The median frequency of $147 \mathrm{~Hz}$ observed for the tiptoe position is of the same order of magnitude as the one predicted theoretically [21]. The results in figure 5 indicate that the shift to lower median frequency occurred during the calf-rising movement thus when the correlation and synchronization increased. This confirms the third hypothesis that if synchronization occurred, the median frequency will drop. This is a strong indication for the increased synchronization of the MUs sensed when the movement becomes more tightly controlled.

\section{EMG decomposition into in-phase and reverse-phase components}

The EMG-signals of neighboring electrodes can be decomposed into an in-phase and a reverse-phase component by projecting the signals on the diagonal vectors as stated in hypothesis four (Figure 2). As expected, the rel-sync-power was higher during the calf-rising movement than during the tiptoe position. This increase in power was attributed to the additional synchronization that was required during the calfrising movement. However, one has to be careful with the interpretation of the absolute values because the power increases non-linearly when MUs get synchronized. A simple computation shows that the power of two separate MUAP is twice the power of the individual MUAP, however, if the two MUAP overlap, the power is four times the power of the individual MUAP. Generally, the power of randomly distributed MUAP is additive but if they are ordered the power is not additive [22]. Thus the rel-syncpower cannot be linearly related to the number of MUs that were synchronized.

\section{Thoughts of caution and limitations}

The whole concept of measuring monopolar EMG-currents is very new and therefore the acquired experience cannot compete with the knowledge that was gained over decades of EMG-potential measurements. The main unknown is how charges that are released or return to the muscle fiber through the ion channels distribute temporarily in the connective tissue. Surely the volume conductor model is too simple because it does not describe any long range effects and does not consider the actual inhomogeneous structure of the muscle seen in the ultrasound images. It does also not consider the local ground potential distribution caused by the electrodes. The purpose of this manuscript was not to provide a full theory of the flow of currents inside the whole muscle. But a few thoughts may help discuss and understand the limitation. A current that flows from any point within the muscle to the reference electrode can either flow through the various electrodes or through the tissue of the body directly to the ground electrode. Thus how the currents distribute themselves between the various pathways to ground potential largely depends on the resistivity between the source and the different electrodes, including the ground electrode. If we assume a homogeneous conductivity in the 
muscle then the distribution is dominated by the distances and cross sectional areas. Thus we can expect that the electrode closest to the source gets most of the current. Based on this view the measured currents reflect the currents from local sources that are located close to the electrodes. Furthermore how the signal depends on the asymmetric placement of the endplates is unknown, it might, as seen in the outliers, cause reversal of the polarity and further research is needed to fully understand the in- and reverse-phase signals. However, we cannot exclude that conductivity is inhomogeneous and thus a certain amount of the in-phase signal is caused by such internal current distributions. If this would be the dominant effect the signals would always be highly correlated; a feature that was not observed in this study. Thus further research is needed to clarify the influence of inhomogeneous intra muscular conductivity.

\section{Conclusions}

The ability to measure the effects of synchronized MUs is essential to understand a coordinated movement. It represents an additional essential variable that can be used in orthopedic assessments of osteoarthritis and other neuromuscular degenerations of joints [23]. This synchronization is often needed during sportive activities and when joint stability plays an essential role e.g. for patients suffering from a lack of stability. These activities often require a strong muscle activity for a short duration of time. The new method of measuring and analyzing monopolar EMG-currents instead of EMG-potentials yields the methodology for observing features of synchronization. The presentwork is a first introduction to the possibilities of measuring the correlation of raw EMG-current signals and applying decomposition into a reverse- and an in-phase component of two EMG-signals. EMG-currents contain information about the power and the frequency properties required to study the effects of synchronization during commonly practiced movements.

\section{References}

1. von Tscharner V, Valderrabano V, Goepfert B (2010) Images of electromyograms that allow digital image processing to resolve muscle activation while exercising or in osteoarthritis. Sport OrthoTrauma 26: 84-88.

2. von Tscharner V, Barandun M, Stirling LM (2011a) Piper rhythm of the electromyograms of the abductor pollicis brevis muscle during isometric contractions. J Electromyogr Kinesiol 21(1): 184-189.

3. von Tscharner V, Barandun M, Stirling LM (2011b) Fatigue-related decrease in Piper rhythm frequency of the abductor pollicis brevis muscle during isometric contractions. J Electromyogr Kinesiol 21(1): 190-195.

4. Stirling LM, von Tscharner V, Kugler P, Nigg BM (2011) Piper rhythm in the activation of the gastrocnemius medialis during running. J Electromyogr Kinesiol 21(1): 178-183.

5. Maurer C, von Tscharner V, Nigg BM (2013) Speed-dependent variation in the Piper rhythm. J Electromyogr Kinesiol 23(3): 673-678.

6. Andreassen S, Rosenflack A (1980) Regulation of the firing pattern of single motor units. J Neurol Neurosurg Psychiatry 43: 897-906.

7. von Tscharner V, Valderrabano V (2010) Classification of multi muscle activation patterns of osteoarthritis patients during level walking. J Electromyogr Kinesiol 20: 676-683.

8. Hodson-Tole EF, Wakeling JM (2009) Motor unit recruitment for dynamic tasks: current understanding and future directions. J Comp Physiol B 179: 57-66.

9. Vieira TM, Loram ID, Muceli S, Merletti R, Farina D (2012) Recruitment of motor units in the medial gastrocnemius muscle during human quiet standing: is recruitment intermittent? What triggers recruitment? J Neurophysiol 107(2): 666-676.

10. Loeb GE (1985) Motoneurone task groups: coping with kinematic heterogeneity. J Exp Biol 115: 137-146.

11. De Luca CJ, Roy AM, Erim Z (1993) Synchronization of motor-unit firings in several human muscles. J Neurophysiol 70(5): 2010-2023.

12.Wakeling JM (2009) The recruitment of different compartments within a muscle depends on the mechanics of the movement. Biol Lett 5(1): $30-34$

13.von Tscharner V, Maurer C, Ruf F, Nigg BM (2013) Comparison of electromyographic signals from monopolar current and potential amplifiers derived from a penniform muscle, the gastrocnemius medialis. J Electromyogr Kinesiol 23(5): 1044-1051.

14. Muramatsu T, Muraoka T, Kawakami Y, Fukunaga T (2002) Superficial aponeurosis of human gastrocnemius is elongated during contraction: implications for modeling muscle-tendon unit. J Biomech 35(2): 217 223.

15. Vieira TM, Loram ID, Muceli S, Merletti R, Farina D (2011) Postural activation of the human medial gastrocnemius muscle: are the muscle units spatially localised? J Physiol 589(2): 431-443.

16. Ashaolu JO, Oni-Orisan OA, Ukwenya VO, Opabunmi OA, Ajao MS (2013) The quadriceps gastrocnemius muscle. Surg Radiol Anat.

17. Dekhuijzen AJ, van Koetsveld PA, Baan GC, Woittiez RD, Huijing PA (1986) Motor endplate position of rat gastrocnemius muscle. Muscle Nerve 9(7): 642-647.

18. Lago P, Jones NB (1977) Effect of motor-unit firing time statistics on e.m.g. spectra. Med Biol Eng Comput 15(6): 648-655.

19. Weytjens JL, van Steenberghe D (1984) The effects of motor unit synchronization on the power spectrum of the electromyogram. Biol Cybern 51(2): 71-77.

20. Merletti R, Lo Conte L, Avignone E, Guglielminotti P (1999) Modeling of surface myoelectric signals--Part I: Model implementation. IEEE Trans Biomed Eng 46(7): 810-820.

21. Dimitrova NA, Dimitrov GV, Nikitin OA (2002) Neither high-pass filtering nor mathematical differentiation of the EMG signals can considerably reduce cross-talk. J Electromyogr Kinesiol 12(4): 235246.

22.von Tscharner V (2010) Amplitude cancellations in surface EMG signals. J Electromyogr Kinesiol 20(5): 1021-1022.

23. Valderrabano V, von Tscharner V, Nigg BM, Hintermann B, Goepfert B, et al. (2006) Lower leg muscle atrophy in ankle osteoarthritis. J Orthop Res 24(12): 2159-2169. 